\title{
Efecto pediculicida de una formulación en base a Eucaliptus globulus L
}

\author{
Marcia Avello, Pola Fernández, Marcos Fernández, Berta Schulz, Marta De Diego, \\ Sigrid Mennickent, Pedro Novoa y Edgar Pastene
}

\section{Pediculicide effect of a Eucaliptus globulus L formulation}

Introduction: Pediculosis capitis is a public health problem with a high prevalence. The emergence of parasite resistance to conventional pediculicide is of great concern worldwide. Objective: To develop alternatives pediculicide, effective and safe, based on the essential oil of Eucaliptus globulus. Method: Through bioassays active concentrations ranges of the essential oil were established, and proceeded to develop a standardized, stable, pharmaceutical form, evaluating its effects on our population. Results: The results showed $100 \%$ effectiveness; short time of death, ovicidal action, activity on the adhesion of the egg, and low toxicity. Discussion: In addition to great effect, the inability of the parasite to become resistant to the chemical composition of the essential oil makes this formulation an alternative to the problem of head lice solution.

Key words: Pediculosis capitis, pediculicide, Eucaliptus globulus.

Palabras clave: Pediculosis capitis, pediculicida, Eucaliptus globulus.

\section{Introducción}

\section{L}

a pediculosis capitis es un problema de salud pública cosmopolita que ha afectado a la humanidad a lo largo de toda su historia. Las frecuencias varían año tras año, constituyéndose en una de las enfermedades parasitarias más frecuentes de la infancia. Su principal forma de transmisión persona a persona, es vía cabeza a cabeza, lo cual se potencia con el contacto cercano y el hacinamiento. En Chile, esta infestación afecta a más de $15 \%$ de la población general, y más de $30 \%$ de la población infantil, principalmente en ambientes escolares ${ }^{1}$.

El agente etiológico, Pediculus capitis humanus, es un insecto que vive sobre el cuero cabelludo y cabellos del ser humano. Su ciclo reproductivo es complejo lo que aumenta la dificultad para erradicarlo ${ }^{2,3}$. Además, la mayoría de los fracasos de tratamiento y persistencia o recidivas de la pediculosis se deben a tratamientos mal aplicados y/o a re-infestaciones: habitualmente porque no se trató el grupo familiar completo, no se realizó extracción de liendres, el pediculicida no fue aplicado en la forma adecuada o por el tiempo adecuado. O simplemente, porque no se dio aviso en el establecimiento escolar y rápidamente se producen re-infestaciones por los compañeros escolares no tratados.

Actualmente, en muchos países, la base del tratamiento de la pediculosis es el uso de insecticidas tópicos, como las piretrinas, deltametrinas, etc.; sin embargo, la resistencia a estos pediculicidas, particularmente la piretrina, ha ido en aumento. En Chile no hay estudios que midan resistencia farmacológica de productos y alternativas terapéuticas disponibles en el mercado. La falta de eficacia de los actuales pediculicidas, así como también, el aumento de la resistencia hacia los productos actualmente disponibles, ha llevado a la urgente necesidad de desarrollo de nuevas alternativas pediculicidas, más efectivas y seguras, que permitan obtener mejores opciones de tratamiento ${ }^{4}$. El desarrollo de nuevas formulaciones para el tratamiento de la pediculosis es un desafío importante para el control de poblaciones resistentes de $P$. capitis a los pediculicidas convencionales ${ }^{5}$. Se requiere de formulaciones eficaces, estables, con óptima biodisponibilidad, buena tolerancia dérmica y libre de efectos tóxicos ${ }^{6,7}$.

Derivados de plantas ampliamente utilizados en medicina tradicional, como los aceites esenciales, se han estudiado con fines pediculicidas. Estos aceites están compuestos por numerosos terpenos, muy volátiles, de bajo peso molecular, que han mostrado una acción comparable a grandes pediculicidas como el lindano y permetrinas, presentando además una acción larvicida, pero con baja toxicidad, vislumbrándose así, como tratamientos alternativos frente a parásitos que han adquirido resistencia frente a fármacos convencionales.

El mecanismo de acción que poseen los aceites esenciales está dado por los terpenos y otros componentes que cumplen un rol antimicrobiano e insecticida fundamental dada su naturaleza lipofílica. Estos componentes interaccionan con las barreras lipídicas de los microorganismos desestabilizando la integridad de las membranas y otras estructuras ricas en moléculas de lipopolisacáridos y asociadas a enzimas, las que son capaces de romper las
Universidad de Concepción. Facultad de Farmacia Departamento de Farmacia.

Los autores declaran no poseer conflictos de interés.

Fuente de Financiamiento: Proyecto INNOVA Chile

12IDL1-13131. Línea 1 Perfil de I+D Aplicada. CORFO.

Solicitud de Patente No 0775-2015

"Formulación Pediculicida en Base

a Aceite Esencial de Eucaliptus

globulus L.", presentada el 27-03-2015.

Recibido: 16 de octubre de 2015 Aceptado: 22 de mayo de 2016

Correspondencia a: Marcia Avello L. maavello@udec.cl 
moléculas introducidas desde el exterior. Se ha propuesto también, la formación espontánea de complejos entre moléculas terpenoides y colesterol de membrana, por ejemplo, lo que causaría un incremento en la permeabilidad de la membrana permitiendo la circulación de iones y macromoléculas entre proteínas. Como consecuencia, se extraerían esteroles a través de vesículas provocando la muerte por falla funcional en el organismo invasor ${ }^{6,7}$.

Uno de los aceites esenciales con más antecedentes de efectividad sobre $P$. humanus es el de Eucaliptus globulus (Eucaliptus), que presenta una importante actividad fumigadora contra $P$. humanus resistentes a permetrina. Dentro de los compuestos activos identificados en el aceite esencial de hojas de Eucaliptus, se ha encontrado 1,8-cineol, (-) $\alpha$-pineno, 2- $\alpha$-pineno, $(E)$-pinocarveol, $l$-felandreno, $\alpha$-terpineno, y 1- $\alpha$-terpineol, compuestos que serían tan efectivos como pediculicidas utilizados normalmente en la terapéutica, como son el ð-fenotrina $\mathrm{y}$ el piretro.

El objetivo de este estudio fue desarrollar una forma farmacéutica pediculicida, en base aceite esencial de E. globulus, estandarizada, estable y con antecedentes de efectividad y seguridad evaluados en una población representativa.

\section{Materiales y Métodos}

\section{Material vegetal y caracterización química}

Las partes aéreas de E. globulus fueron recolectadas en los bosques del campus de la Universidad de Concepción. La identificación botánica se realizó en el Departamento de Botánica, Facultad de Ciencias Naturales y Oceanográficas, Universidad de Concepción.

La obtención de aceite esencial de E. globulus se realizó a través de hidrodestilación en aparato Clavenger. Se sumergieron $800 \mathrm{~g}$ de hojas y partes aéreas frescas en 5 litros agua, durante $12 \mathrm{~h}$. Luego se llevó a ebullición por 90 min. El aceite esencial obtenido se secó con sulfato de sodio anhidro y se almacenó refrigerado a $4^{\circ} \mathrm{C}$ en un recipiente ámbar, hasta el momento de su utilización. El rendimiento se determinó como $\mathrm{ml}$ de aceite esencial obtenido por cada $100 \mathrm{~g}$ de planta utilizada. La caracterización química se efectuó por cromatografía de gases.

\section{Bioensayos y estudios de toxicidad in vitro}

Con el fin de comprobar la eficacia del extracto frente a $P$. humanus, se realizaron ensayos in vitro. Un promedio de cuatro parásitos en sus formas adultas y jóvenes (huevos) se depositaron en cápsulas de vidrio con cabello humano. Se expusieron a distintas diluciones de aceite esencial en mezclas alcohol agua. La mortalidad de los parásitos adultos se evaluó cada minuto hasta observar cambios durante una hora. Se consideraron muertos cuando sus apéndices dejaron de moverse estimulados con una tórula o pérdida del reflejo de enderezamiento. En los huevos, se evaluó una vez al día por 7 días. Se consideraron muertos cuando a los 7 días no se observó eclosión ${ }^{11}$. Como control se usó deltametrina 0,02 g y piperonil butóxido 2,5 g, loción.

\section{Desarrollo y caracterización de la formulación}

Se desarrolló una formulación tipo loción hidroalcohólica en base con aceite esencial de E. globulus. A la formulación se le realizaron controles in vitro tales como viscosidad; pérdida de agua por evaporación; tamaño y distribución de tamaño de partícula; cantidad y liberación de principios activos mediante el uso de celdas de Franz y estudio de estabilidad. En el estudio de estabilidad se controló la potencia del producto (estabilidad química) y características físicas. Se evaluó la concentración de un marcador químico a $0,30,90$ y 180 días para determinar si se encontraba dentro de los límites aceptables. Un cambio significativo del producto corresponde a un cambio de 5\% en la concentración inicial de principio activo y a cambios en los atributos físicos (apariencia) y test de funcionalidad (color, separación de fases, dureza, etc.) (Conferencia Internacional en Armonización $\mathrm{ICH}$ ).

\section{Bioensayos y estudios de toxicidad in vitro de la formulación}

Los bioensayos se desarrollaron para evaluar la efectividad de la formulación frente a parásitos adultos y jóvenes (huevos). Estos fueron "toxicidad en fase vapor" y "toxicidad por inmersión". Para evaluar la seguridad se efectúo la "toxicidad sobre tejido" (ensayo HETCAM $)^{12-15}$. Como control se usó deltametrina 0,02 g y piperonil butóxido 2,5 g, shampoo.

\section{Ensayo piloto abierto y aleatorizado}

Se inspeccionó establecimientos educacionales de la ciudad de Concepción en busca de niños con síntomas relacionados con pediculosis capitis. Una vez detectado un brote de pediculosis, se procedió a identificar a los posibles participantes del estudio. Se citó a los padres a una charla informativa, donde se les explicó en qué consistía el estudio, y sus responsabilidades en éste. Se procedió a realizar la intervención sólo en aquellos niños que quisieron participar del estudio, y que contaban con un consentimiento informado firmado y una autorización para formar parte del estudio, firmado por sus padres.

Los participantes fueron seleccionados por inspección visual por tres minutos al encontrarse al menos $25 \mathrm{P}$. humanus en el cuero cabelludo. Los criterios de exclusión de los participantes fueron: uso de productos pediculicidas, antihelmínticos o antimicrobianos dentro de las cuatro semanas previas al tratamiento; desórdenes graves en la piel del cuero cabelludo, tales como dermatitis, eccema, 
procesos irritativos, o cualquier alteración que pudiese aumentar la absorción del producto, o la gravedad del cuadro de base; tratamiento cosmético del cabello dentro de las cuatro semanas previas al tratamiento; sensibilidad a cualquier ingrediente del producto; enfermedad mental.

El estudio piloto se realizó finalmente con 10 niños infestados de edades entre 5 a 15 años, que contaban con las autorizaciones correspondientes.

Los niños en estudio fueron distribuidos por un método de aleatorización simple según número de registro en el estudio, de la siguiente forma; los impares se asignaron a un grupo control, en tratamiento con pediculicida registrado de amplio uso, (5 voluntarios), y los pares al grupo de intervención en tratamiento con el producto innovador (5 voluntarios).

Cada tutor recibió las indicaciones para la adecuada aplicación del producto. Se les solicitó, a su vez, la limpieza de los artículos personales, de vestir y de aseo, de los participantes y contactos directos, con agua hirviendo o calor seco, con el fin de evitar la reinfestación. El tratamiento de los contactos directos fue el mismo recibido por el grupo control.

Para el estudio se utilizó un producto comercial de alta salida, ampliamente utilizado para el manejo de estas infestaciones, constituido por deltametrina $0,02 \mathrm{~g}$ y piperonil butóxido 2,5 g, en loción; el producto innovador utilizado fue una loción en base a eucaliptus al 5\%

El esquema de utilización fue el mismo tanto para el producto en estudio, como para el producto comercial. Los participantes recibieron una primera aplicación por 30 min, repitiéndola a los 7 días de tratamiento. Los productos fueron aplicados generosamente en la totalidad del cuero cabelludo del paciente, cubriendo las raíces del pelo y sobre todo detrás de las orejas y cuello, sobre pelo seco. Después del tiempo de exposición se lavaron el cuero cabelludo con abundante agua tibia. Una vez terminado el proceso, se debió peinar el pelo para la eliminación de las liendres, para su posterior observación en el laboratorio. Se realizó una inspección al segundo, séptimo día de aplicación y dos días después de terminado el tratamiento, en busca de piojos vivos, liendres o huevos. Las inspecciones fueron realizadas siempre por el mismo investigador, y duraron de 3 a 5 min con apoyo de una lupa. Para evaluar la respuesta a tratamiento se consideró el grado de curación, mediante la asignación de un puntaje en base a la cantidad de especímenes encontrados (Tabla 1). Se consideró el tratamiento efectivo a la completa ausencia de $P$. humanus vivos sobre el cuero cabelludo húmedo uno o dos días después de la primera aplicación, y a la completa ausencia de huevos dos días después de terminado el tratamiento.

Se consideraría falla al tratamiento con puntajes igual o mayor a 1 .

Se consideró muerte del piojo si este era incapaz de caminar sobre un papel filtro, permanecía acostado sobre su espalda, o no presentaba movimiento de sus antenas o patas después de un período de $18 \mathrm{~h}$. El criterio de mortalidad de los huevos fue encontrar huevos sin eclosionar, o huevos eclosionados con la ninfa en el interior.

Para medir la seguridad del producto, se registró antes, durante, y después de aplicado el tratamiento, el grado de picazón, a través de la escala visual análoga, y la eventual aparición de efectos adversos.

La aceptación cosmética del producto, se evaluó mediante un cuestionario que incluyó características organolépticas del producto, irritación del cuero cabelludo y cambios cosméticos del cabello. Dentro de las variables medidas se consideró enrojecimiento, sequedad, irritación, descamación y molestia, se utilizó una escala de 0 a 3 , donde 0 correspondía ausencia de sintomatología y 3 presencia severa ${ }^{16,17}$.

Este estudio fue aprobado por el Comité de Ética de la Universidad de Concepción.

\section{Resultados}

\section{Material vegetal y caracterización química}

Se obtuvo aceite esencial de partes aéreas de E. globulus con un rendimiento de 1,43 $\pm 0,05(\mathrm{ml} / 100 \mathrm{~g} \pm \mathrm{DE})$. En el análisis cromatográfico se obtuvo un perfil con la aparición de 4-5 compuestos principales (Tabla 2), los que corresponden en identidad y abundancia a lo descrito para esta especie.

Tabla 1. Asignación de puntaje según número de especímenes encontrados

\begin{tabular}{lcc}
\hline Clasificación & n de especímenes & Puntaje asignado \\
No se encuentran liendres & 0 & 0 \\
Infestación leve & $<$ de 10 liendres & 1 \\
Infestación moderada & Entre 10 a 20 liendres & 2 \\
Infestación severa & $>20$ liendres & 3 \\
\hline
\end{tabular}

Tabla 2. Compuestos identificados en el aceite esencial de $E$. globulus y su porcentaje

\begin{tabular}{|cr|}
\hline Compuesto & \multicolumn{1}{c}{$\%$} \\
\hline$\alpha$-pineno & $0,05-10,0$ \\
\hline$\beta$-pineno & $0,05-1,5$ \\
\hline Sabineno & Máximo 0,3 \\
$\alpha$-fellandreno & $0,05-1,5$ \\
\hline Limoneno & $0,05-15,0$ \\
1,8 cineol & Mínimo 70,0 \\
\hline Alcanfor & Máximo 0,1 \\
\hline
\end{tabular}


Tabla 3. Tiempo de muerte provocado por el aceite esencial de $E$. globulus

\begin{tabular}{|c|c|c|c|c|}
\hline \multirow[t]{5}{*}{ E. globulus } & $4 \%$ & 4 (P. humanus) & 2 (muertos) & 1,5 (tiempo) \\
\hline & & & 2 (muertos) & 2,5 (tiempo) \\
\hline & $5 \%$ & 11 (P. humanus) & 4 (muertos) & (tiempo) \\
\hline & & & 2 (muertos) & (tiempo) \\
\hline & & & 5 (muertos) & 3 (tiempo) \\
\hline
\end{tabular}

Todos los aceites esenciales fueron disueltos en $\mathrm{EtOH} 50 \%(100 \mu \mathrm{l})$.

Figura 1. Efectividad (\%) \& tiempo (min) in vitro de la loción hidroalcohólica.

\begin{tabular}{|lc|}
\hline \multicolumn{2}{|c|}{ Tabla 4. Formulación tipo loción hidroalcohólica } \\
\hline Materia prima & Concentración (\%v/v) \\
Etanol $96^{\circ}$ & $32,5 \%$ \\
Isopropanol & $32,5 \%$ \\
Aceite esencial de E. globulus & $5 \%$ \\
Agua destilada & $30 \%$ \\
\hline
\end{tabular}

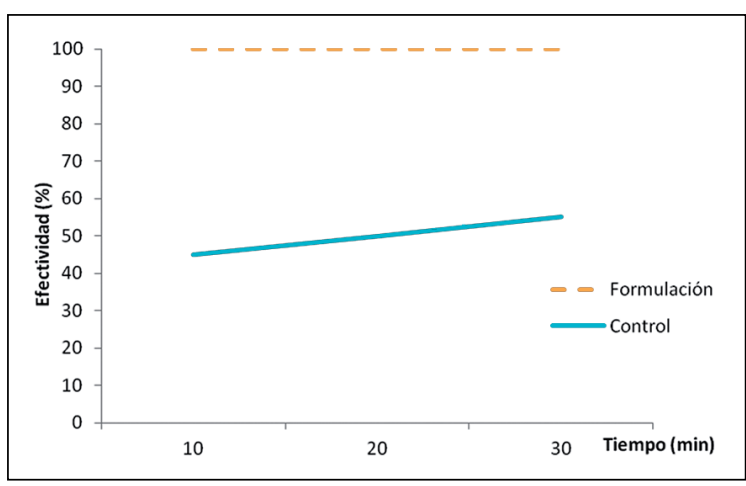

\section{Bioensayos y estudios de toxicidad in vitro}

Se observó un $100 \%$ de efectividad pediculicida y ovicida con aceite esencial de E. globulus 5\% (Tabla 3). El producto control utilizado atenuaba las reacciones de los parásitos manteniéndolos inmóviles por $10 \mathrm{~min}$, luego de los cuales se reanimaban. Eucaliptus globulus actúo, además, en menos tiempo que el control (1-3 min). Con respecto a la actividad ovicida, no se observó eclosión a los 7 días.

\section{Desarrollo y caracterización de la formulación}

La formulación desarrollada (Tabla 4) cumple con los controles de rutina y mantiene sus características físico-organolépticas y la concentración del marcador químico elegido (1,8-cineol, 44,8-45\%) durante el tiempo de estudio en condiciones a tiempo real y en condiciones aceleradas. Se utilizó 5\% de aceite esencial de E. globulus por ser la concentración más activa detectada en los bioensayos.

\section{Bioensayos y estudios de toxicidad in vitro de la formulación}

En la Figura 1 se observa una actividad fumigante de la loción hidroalcohólica de $100 \%$, medida a los $10 \mathrm{~min}$. La mortalidad de los huevos tratados (100\%) se registró después de cinco días de la eclosión del control.

No se observó irritación con la formulación.

\section{Ensayo piloto}

El porcentaje de pacientes sanos después de la aplicación del tratamiento fue de $100 \%$ con un puntaje de (0), es decir, no se encontraron liendres durante la inspección; el grupo control en cambio, presentó un puntaje de (3), correspondiente a más de 20 liendres. El nivel de infestación fue "severo" para los dos grupos de tratamiento.

Al medir la seguridad de la formulación, se obtuvo para todos los parámetros en estudio un puntaje total de 0 , correspondiente a ausencia de enrojecimiento, sequedad, irritación y descamación, al igual que el grupo que utilizó el producto comercial.

En relación a la aceptabilidad cosmética del preparado, evaluada mediante un cuestionario estándar, se obtuvo a su vez un puntaje de 0 , correspondiente a ausencia de molestia, irritación y picazón, al igual que el grupo control.

\section{Discusión}

La presente tecnología corresponde a una formulación innovadora con efecto pediculicida en base a aceite esencial E. globulus. Considera y cumple con las normas de calidad, estandarización y pruebas de efectividad en humanos.

Si bien la pediculosis humana es una condición muy común en el mundo, pocos grupos de investigadores han experimentado alternativas a su tratamiento. Existen antecedentes que indican actividad pediculicida de compuestos volátiles de naturaleza terpénica y fenólica, usualmente concentrados en plantas aromáticas introducidas, pero que se encuentran dentro de productos de amplia distribución y comercialización nacional por su uso como especias ${ }^{18}$. Estos extractos han mostrado una acción comparable a grandes pediculicidas como el lindano, permetrinas y con acción larvicida, pero con baja toxicidad, vislumbrándose como tratamientos alternativos frente a parásitos que han adquirido resistencia frente a fármacos convencionales ${ }^{19,20}$.

La innovación de esta formulación considera: extracto con actividad ovicida, los aceites esenciales, por sus mecanismos moleculares sobre las membranas biológicas y organelos celulares que tendrían efectos deletéreos sobre las formas adultas y huevos del P. humanus, causando la muerte del parásito, en ambos estados; actividad sobre la adherencia del huevo $P$. humanus, los aceites esenciales, por sus características de lipofilicidad tienen además, acción sobre la sustancia adherente del huevo sobre el 
cuero cabelludo solubilizándola; corto tiempo de muerte, la sinergia de mecanismos de acción entre los distintos tipos de principios activos estaría favoreciendo a que el tiempo de muerte del parásito sea menor; nula toxicidad, los efectos adversos se ven minimizados con el manejo de la dosis; imposibilidad del parásito de adquirir resistencia, el mecanismo sobre los lípidos de las membranas biológicas imposibilita al parásito de adquirir resistencia; amplia distribución de aceites esenciales en la naturaleza, los extractos de aceites esenciales comparten propiedades biológicas en común, por lo tanto, se pueden considerar potenciales materias primas otras fuentes de aceites esenciales, evaluando su disponibilidad.

Como otros aspectos distintivos, cabe destacar la administración del extracto en una forma farmacéutica adecuada para la administración capilar. La formulación, al estar presentada en forma de loción, permite una fácil y eficiente extensión en zonas de alta densidad capilar. Es una forma farmacéutica líquida que permite que el tiempo de contacto entre el preparado pediculicida y el parásito, sea más prolongado que el que ofrecen otras formas de aplicación. Permite por lo tanto, una mayor penetración, una mayor actividad residual, confiriendo un poder ovicida a la formulación, mejor que las cremas. Estas ca- racterísticas hacen que las lociones sean consideradas las formas de elección en el tratamiento de estas parasitosis.

Se considera repetir el estudio piloto usando un tamaño de muestra mayor para validar las conclusiones con mayor evidencia.

\section{Resumen}

Introducción: La pediculosis capitis es un problema de salud pública con una alta prevalencia. La aparición de resistencia del parásito a los pediculicidas convencionales es de gran preocupación a nivel mundial. Objetivo: Desarrollar alternativas pediculicidas, efectivas y seguras, en base al aceite esencial de Eucaliptus globulus. Método: A través de bioensayos se establecieron rangos de concentraciones activas del aceite esencial, y se procedió al desarrollo de una forma farmacéutica, estandarizada, estable, evaluando sus efectos en nuestra población. Resultados: Los resultados mostraron $100 \%$ de efectividad; corto tiempo de muerte, acción ovicida, actividad sobre la adherencia del huevo, y baja toxicidad. Discusión: Además de la gran efectividad, la imposibilidad del parásito de adquirir resistencia a la composición química del aceite esencial hace de esta formulación una solución alternativa al problema de la pediculosis.

\section{Referencias bibliográficas}

1.- Moreno M C. Ectoparasitosis de importancia en Chile: Epidemiología y terapia. Rev Chilena Infectol 2011; 28: 435-9.

2.- Cazorla D, Ruiz A, Acosta M. Clinical and epidemiological study of pediculosis capitis in schoolchildren from Coro Venezuela. Invest Clin 2007; 48: 445-57.

3.- Nuttall G H F. The biology of Pediculus humanus. Parasitology 1917; 10 (01): 80-185.

4.- MINSAL: Guía Clínica de Sarna y Pediculosis 2007. [Acceso 28/09/2013].

5.- Burkhart C G. Relationship of treatmentresistant head lice to the safety and efficacy of pediculicides. Mayo Clin Proc 2004; 79 (5): 661-6.

6.- Isman M, Miresmailli S, Machial C. Commercial opportunities for pesticides based on plant essential oils in agriculture, industry and consumer products. Phytochem Rev 2011; 10 (2): 197-204.

7.- Toloza A C, Zygadlo J, Cueto G M. Fumigant and repellent properties of essential oils and component compounds against permethrinresistant Pediculus humanus capitis (Anoplura: Pediculidae) from Argentina. J Med Entomol 2006; 43 (5): 889-895

8.- Choi H Y, Yang Y C, Lee S H, Clark J M, Ahn Y J. Efficacy of spray formulations containing binary mixtures of clove and eucalyptus oils against susceptible and pyrethroid/malathion-resistant head lice (Anoplura: Pediculidae).

J Med Entomol 2010; 47 (3): 387-91

9.- $\quad$ Yang Y C, Choi H Y, Choi W S, Clark J M, Ahn Y J. Ovicidal and adulticidal activity of Eucalyptus globulus leaf oil terpenoids against Pediculus humanus capitis (Anoplura: Pediculidae). J Agr Food Chem 2004; 52 (9): 2507-25.

10.- Toloza A, Lucía A, Zerba E, Masuh H, Picollo M I. Eucalyptus essential oil toxicity against permethrin-resistant Pediculus humanus capitis (Phtiraptera: Pediculidae). Parasitol Res 2010; 106: 409-14

11.- Heukelbach J, Oliveira F S, Speare R. A new shampoo based on neem (Azadirachta indica) is highly effective against head lice in vitro. Parasitol Res 2006; 99 (4): 353-6.

12.- Yang Y C, Lee S H, Choi D H, Ahm Y J. Ovicidal and adulticidal effects of Eugenia caryophyllata bud and leaf oil compounds on Pediculus capitis. J. Agric. Food Chem 2003; 13 (17): 4884-8.

13.- Cueto G M, Audino P G, Vassena C V, Picollo M I, Zerba E N. Toxic effect of aliphatic alcohols against susceptible and permethrinresistant Pediculus humanus capitis (Anoplura: Pediculidae) J Med Entomol 2002; 39 (3): 457-60.

14.- Scheel J, Kleber M, Kreutz J. Eye irritation potential: Usefulness of the HET-CAM under the Globally Harmonized System of
Classification and labeling of chemicals (GHS). Regul Toxicol Pharm 2011; 59 (3): 471-92.

15.- Mougabure C, Gaston V, Claudia G. Efectividad de lociones capilares sobre poblaciones de Pediculus capitis resistentes a insecticidas. Acta Toxicol Argent 2000; 8 (1): 10-2.

16.- Fujii T, Furukawa S, Suzuki S. Studies on compounded perfumes for toilet godos. On the non-irritative compounded perfumes for soaps. Yukagaku 1972; 21: 904-8

17.- Mac-Mary S, Messkh R, Jeudy A, Lihoraeu T, Sainthillier J M, Gabard B, et al. Assessment of the efficacy and safety of a new treatment for head lice. Dermatology 2012; 1-6.

18.- González-Audino P, Vassena C, Zerba E, Picollo, M. Effectiveness of lotions based on essential oils from aromatic plants against permethrin resistant Pediculus humanus capitis. Arch Dermatol Res 2007; 299 (8): 389-92.

19.- Silvestre A J D, Cavaleiro J S, Delmond B, Filliatre C, Bourgeois G. Analysis of the variation of the essential oil composition of Eucalyptus globulus Labill. from Portugal using multivariate statistical analysis. Ind Crop Prod 1997; 6 (1): 27-33.

20.- Tyagi A K, Malik A. Antimicrobial potential and chemical composition of Eucalyptus globulus oil in liquid and vapour phase against food spoilage microorganisms. Food Chem 2011; 126 (1): 228-35. 